Recibido 05 de Enero, 2019 - Aceptado 05 de Febrero, 2019

\title{
Agujeros negros de antimateria
}

\section{Black antimaterial holes}

Enrique Álvarez ${ }^{1}$

\section{RESUMEN}

En el presente trabajo proponemos un modelo gravitatorio alternativo a la teoría de la relatividad general, en el que la curvatura espaciotemporal se modificaría por efecto de la interacción de las partículas virtuales de materia y antimateria entre ellas y la materia, a través de subniveles fractales del vacío cuántico, generando una secuencia de apantallamientos y antiapantallamientos gravitatorios. Se modificarían las ecuaciones de movimiento de los planetas basados en la métrica de Schwarzschild y sus variantes, así como las actuales teorías sobre los agujeros negros que estarían conformados de antimateria.

Palabras claves: Subniveles fractales del vacío cuántico, cargas vectoriales, partículas virtuales, métrica de Schwarzschild modificada, ecuaciones de movimiento de planetas, perihelio de Mercurio, agujeros negros de antimateria.

\section{ABSTRACT}

In this work, we propose an alternative gravity model to the theory of general relativity. In our model, the space-time curvature would be modified by the interaction of virtual particles of matter and antimatter among each other and with matter. This would take place across fractal sub-levels of the quantum vacuum, generating a sequence of gravitational screenings and anti screenings. The equations of motion of the planets based on the Schwarzschild metric and its variants would be modified along with current theories about black holes which would be made of antimatter.

Keywords: Fractal sub-levels of the quantum vacuum, vector charges, virtual particles, modified Schwarzschild's metric, planetary motion equations, Mercury perihelion, antimatter black holes. 


\section{INTRODUCCIÓN}

Antes de introducirnos en la relatividad general abordaremos la mecánica newtoniana como caso límite. Siendo la gravedad nuestro objeto de estudio, consideraremos una masa donde las fuerzas electrostáticas se anulan entre sí por la equiparidad de protones y electrones en átomos o moléculas. La fuerza fuerte se anula en el núcleo del átomo, quedando la gravedad como una fuerza residual de una masa al no encontrar partículas reales de antimateria que la neutralicen.

Analicemos el proceso fractal de una partícula elemental como el electrón, por ejemplo. Este atrae positrones virtuales de antimateria del vacío cuántico que atenúan la carga negativa del electrón, efecto comprobado experimentalmente. En publicaciones anteriores [1], hemos postulado la existencia de subniveles del vacío cuántico conformado por electrones virtuales de un segundo nivel del vacío cuántico que a su vez serían atraídos por los positrones virtuales de un primer nivel del vacío cuántico lo cual atenuaría su efecto. A su vez estos electrones virtuales atraerían positrones virtuales de un tercer nivel que atenuarían también su efecto. Este proceso de interacciones continuaría indefinidamente en una secuencia fractal infinita convergente, neutralizando la carga del electrón a una distancia nula.

La figura 1 nos muestra una representación gráfica del proceso. La partícula real del electrón está representada por una esfera grande de color rojo. Los positrones virtuales del primer nivel del vacío cuántico por esferas menores de color azul alrededor del electrón. Los electrones virtuales del segundo nivel por esferas más pequeñas de color rojo alrededor de los positrones virtuales. La secuencia continúa indefinidamente de modo fractal.

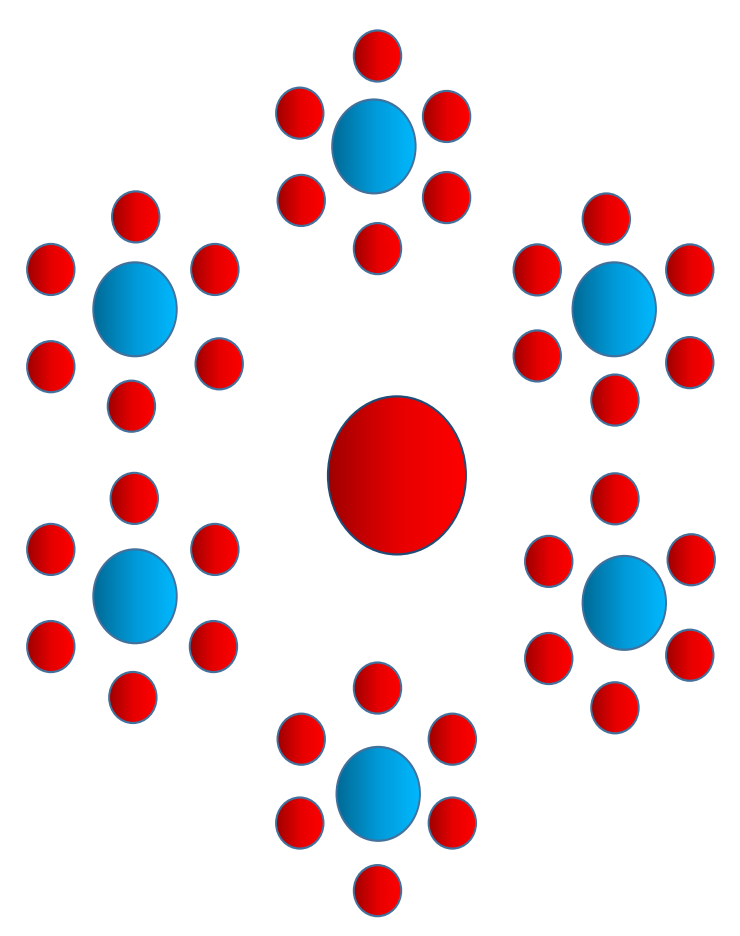

Figura №1. Representación gráfica del proceso de interacción de las partículas virtuales de materia y antimateria en los subniveles del vacío cuántico. 
La ecuación encontrada por nosotros que define la intensidad E de la carga eléctrica estaría dada por [2]:

$$
E=\frac{e^{-}}{4 \pi \epsilon_{0} r^{2}} e^{-\frac{\alpha \hbar}{m_{e} c r}}(0.1)
$$

siendo e- la carga del electrón, $\epsilon_{0}$ la constante de permitividad en el vacío, $r$ la distancia al electrón, a la constante cosmológica, h la constante reducida de Planck, m_e la masa del electrón y c la velocidad de la luz en el vacío.

La figura 2 representa la gráfica de la intensidad E del electrón, lo que explicaría por qué el electrón como partícula puntual no estalla por el efecto de su propia carga repulsiva, sin necesidad de recurrir a la renormalización. El área que encierra esta curva representa la energía del electrón.

Lo mismo ocurriría con los quarks [3]. En este caso, como se aprecia en el gráfico, para distancias cortas, la intensidad aumenta con la distancia, que es precisamente lo que ocurre con los quarks, lo que explicaría el confinamiento de estas partículas y su libertad asintótica.

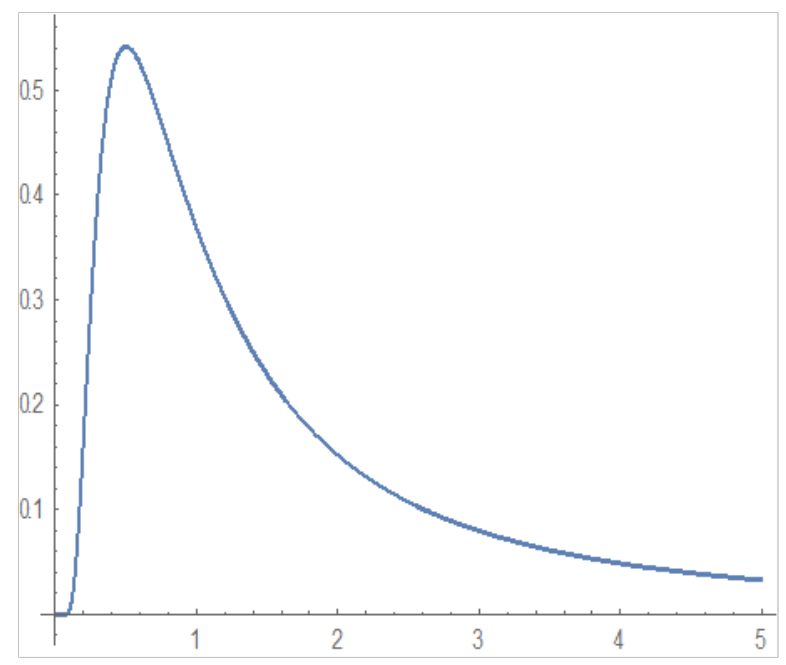

Figura Na 2. Gráfica de la intensidad electrostática del electrón en función de la distancia a la partícula.
Si bien, como acabamos de mencionar, las cargas nucleares fuertes y electrostáticas se anulan entre sí, las partículas virtuales de antimateria se mantienen adheridas a las partículas elementales reales, ejerciendo su influencia antigravitatoria.

\section{Cargas vectoriales.}

En publicaciones anteriores propusimos que las cargas cromodinámicas, electrostáticas y gravitatorias se pueden representar como vectores complejos.

Por ejemplo, las cargas electrostáticas se representan como dos vectores reales unitarios que forman entre sí un ángulo de $0^{\circ}$ si son del mismo signo y $180^{\circ}$ si son de signo contrario.

Las cargas cromodinámicas de un barión se representan como tres vectores reales unitarios y simétricos en un plano, en lugar de colores, que forman entre sí un ángulo de $120^{\circ}$. La suma de los tres vectores es nula, al igual que la suma de los tres colores da blanco.

La carga gravitatoria se representa por el vector imaginario $\boldsymbol{u} e^{i \pi / 2}=\boldsymbol{u} i$. La atracción o repulsión entre una carga y otra se define por el producto escalar complejo de los vectores unitarios y viene dada por [4]:

$$
\text { ui.ui }=e^{i(\alpha+\beta)} \cos \theta \text { (1.1) }
$$

siendo $\theta$ el ángulo que forman los vectores entre sí $y \propto$ y $\beta$ los ángulos que determinan el carácter real o imaginario del vector, correspondiendo la naturaleza real a las cargas electrostáticas y cromodinámicas y la imaginaria a la carga gravitatoria para $\propto=\beta=\pi / 2$

En el caso de dos cargas electrostáticas del mismo signo, es decir, $\alpha=\beta=0$ y $\theta=0^{\circ}$, el producto escalar es +1 y las cargas se repelen. En caso de cargas de diferente signo, $\alpha=\beta=0$ y $\theta=180^{\circ}$, el producto escalar es - 1 y las cargas se atraen. Tratándose de cargas cromodinámicas, donde 
$\alpha=\beta=0 \quad$ y $\theta=120^{\circ}$, se suman los productos escalares de una de las cargas con las dos restantes que da como resultado -1 y las cargas se atraen, que es precisamente el comportamiento de los quarks en los nucleones.

Las cargas gravitatorias donde $\alpha=\beta=\pi / 2$ y $\theta=0^{\circ}$, el producto escalar es -1, es decir, dos masas de materia se atraen, así como dos masas de antimateria también se atraen.

Tratándose de cargas gravitatorias donde $\alpha=\beta=\pi / 2$ y $\theta=180^{\circ}$, el producto escalar es +1 y las cargas se repelen, es decir, una masa de materia y otra de antimateria se repelen [5], resultado importante como veremos más adelante.
Para algunos físicos la materia y antimateria se atraen gravitatoriamente, para otros se repelen. Hasta la fecha no hay ningún experimento que compruebe el comportamiento gravitatorio entre materia y antimateria. Nosotros proponemos que se repelen.

La proximidad de dos nucleones, protones o neutrones, en un átomo, produce una ligera asimetría en cada nucleón, dando lugar a un pequeño vector resultante en ambos nucleones en sentido opuesto uno del otro, representados en color rojo, que es la fuerza residual fuerte que une a los nucleones y neutraliza la carga eléctrica repulsiva de los protones a través de los neutrones, como se ilustra en la figura 3 [6].
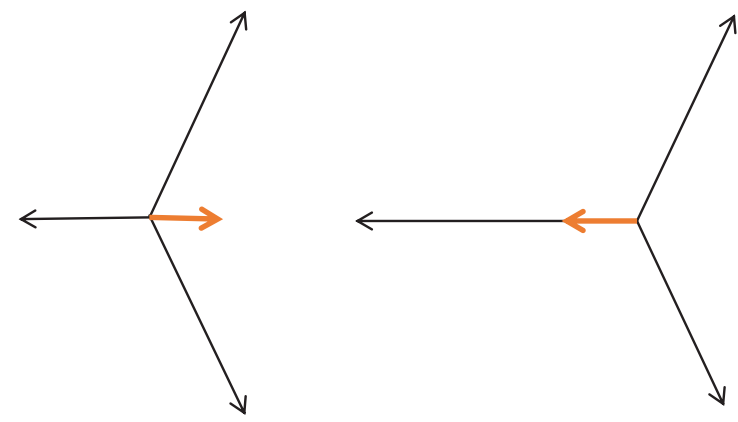

Figura No3. Representación gráfica vectorial de la fuerza residual fuerte entre dos nucleones.

Esto da como resultado un empaquetamiento cúbico de los nucleones en el átomo, que es la que explica la presencia de neutrones que hacen posible la unión de los protones, como se muestra por ejemplo en la figura 4 que representa el átomo de berilio, el isótopo más estable de cuatro protones y cinco neutrones, en el que los protones están representados por las esferas rojas y los neutrones por las azules.

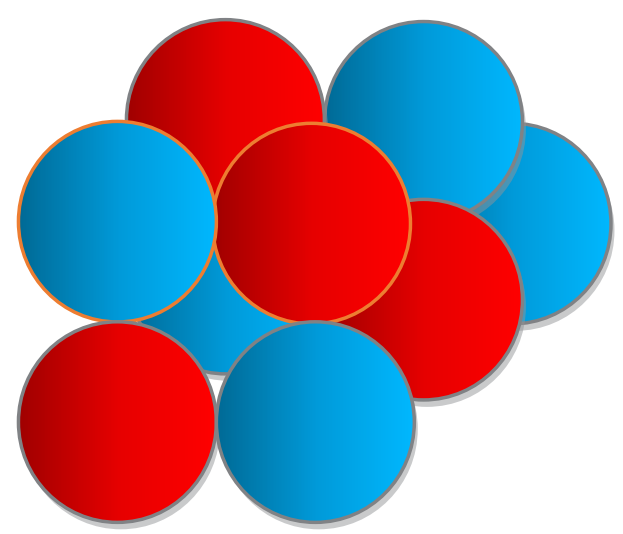

Figura No 4. Gráfica del compactamiento cúbico entre los nucleones del átomo de berilio. 
Las cargas unitarias de las fuerzas fundamentales de la naturaleza pueden representarse en un campo vectorial complejo de cuatro dimensiones, como se ilustra en la figura 5, en el que las cargas de color de los quarks están representadas en el plano conformado por los vectores direccionales $i$, $j$, en el cual definimos por convención las tres cargas de un barión como $\boldsymbol{i}, \quad-\frac{1}{2} \boldsymbol{i}+\frac{\sqrt{3}}{2} \boldsymbol{j}, \quad-\frac{1}{2} \boldsymbol{i}-\frac{\sqrt{3}}{2} \boldsymbol{j} ;$ la carga electrostática por $k$ y la carga gravitatoria por $l i$, siendo $i$ la unidad imaginaria. La gravedad, la única fuerza que no ha sido unificada hasta el día de hoy, poseería una carga imaginaria, así como el campo gravitatorio y la masa gravitatoria $m i$, a diferencia de la masa inercial $m$, que sería de carácter real, concepto no contemplado por la física. En este caso, los vectores direccionales forman un ángulo de $90^{\circ}$ entre sí, cuyo coseno que define el producto escalar es 0 , es decir, no hay atracción ni repulsión, consecuente con el hecho de que estas fuerzas no interactúan entre ellas.

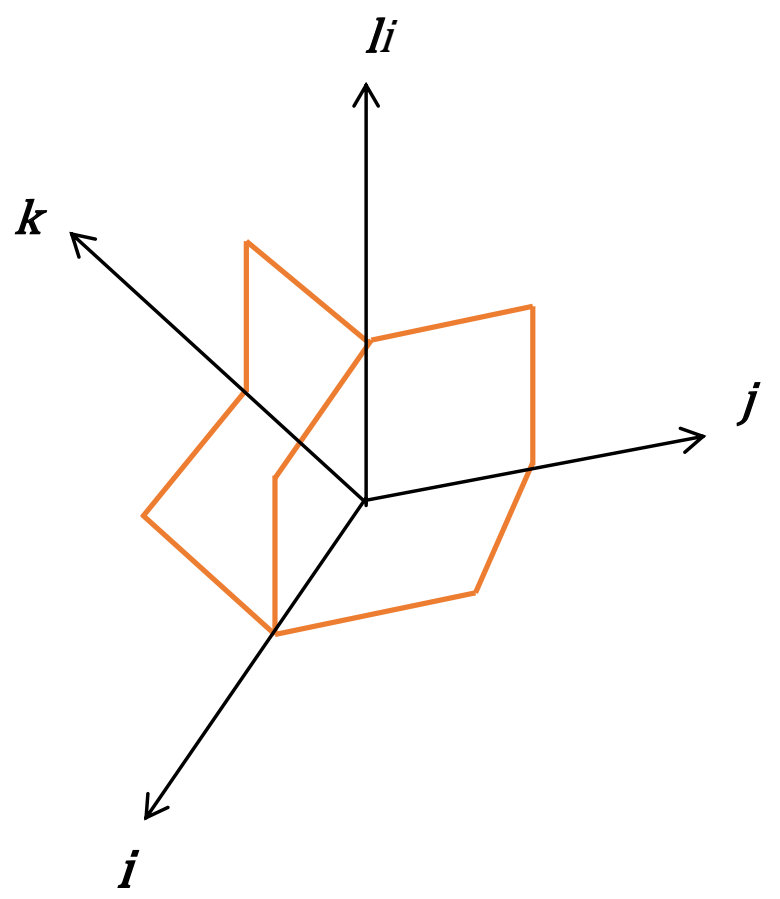

Figura No 5 . Representación de un campo vectorial complejo en cuatro dimensiones de las cargas cromodinámica, electrostática y gravitatoria de una partícula

2. Gravedad newtoniana modificada.

De modo análogo al cálculo realizado por nosotros para determinar la intensidad de la carga eléctrica y energía del electrón en una publicación anterior [7], procederemos con la gravedad newtoniana modificada. Consideremos una masa $M$ de simetría esférica y estática. La fuerza de atracción gravitatoria que la masa ejerce sobre sí misma en el cascarón esférico de radio $r$ está dada por:

$$
\boldsymbol{F}=-\frac{G M^{2}}{r^{2}} \boldsymbol{r}
$$


siendo $G$ la constante de gravitación y $r$ un vector unitario orientado hacia el centro de $M$; el signo negativo indica la orientación de la fuerza dirigida hacia el centro de $M$. La energía potencial gravitatoria $E_{0}$ entre dos puntos $a$ y $b$ está dada por:

$$
E_{0}=\int_{a}^{b}-\frac{G M^{2}}{r^{2}} \cdot d r=-G M\left(\frac{1}{a}-\frac{1}{b}\right)
$$

Si multiplicamos y dividimos la ecuación por $\lambda$, una longitud vinculada a $M$, por ahora indeterminada, tendremos:

$$
E_{0}=-\frac{G M^{2}}{\lambda}\left(\frac{\lambda}{a}-\frac{\lambda}{b}\right)
$$

Hagamos $\lambda / r=\phi_{r}$, una función de $r$, de manera que:

$$
E_{0}=-\frac{G M^{2}}{\lambda}\left(\phi_{a}-\phi_{b}\right)
$$

Obtenemos así la energía gravitatoria en función de una nueva variable $\phi_{r}$. De manera análoga, de acuerdo a nuestra hipótesis, la energía $E_{1}$ generada por los positrones virtuales en función de $\phi_{r}$ estaría dada por:

$$
E_{1}=\int_{\phi_{a}}^{\phi_{b}}-\frac{G M^{2}}{\lambda}\left(\phi_{a}-\phi_{b}\right) \cdot d \phi=\frac{G M^{2}}{\lambda}\left(\frac{\phi_{a}^{2}}{2 !}-\frac{\phi_{b}{ }^{2}}{2 !}\right)
$$

El cambio de signo se debe a que la energía gravitatoria de los positrones es positiva. Del mismo modo, la energía gravitatoria de los electrones del segundo nivel del vacío cuántico estaría dada por:

$$
E_{2}=\int_{\emptyset_{a}}^{\emptyset_{b}} \frac{G M^{2}}{\lambda}\left(\frac{\phi_{a}{ }^{2}}{2 !}-\frac{\phi_{b}{ }^{2}}{2 !}\right) \cdot d \phi=-\frac{G M^{2}}{\lambda}\left(\frac{\phi_{a}{ }^{3}}{3 !}-\frac{\phi_{b}{ }^{3}}{3 !}\right)
$$

En general, la energía gravitatoria de un nivel $n$ del vacío cuántico estaría dada por:

$$
E_{n}=\int_{\phi_{a}}^{\phi_{b}}(-1)^{n} \frac{G M^{2}}{\lambda}\left(\frac{\emptyset_{a}{ }^{n}}{n !}-\frac{\emptyset_{b}{ }^{n}}{n !}\right) \cdot d \emptyset=(-1)^{n+1} \frac{G M^{2}}{\lambda}\left(\frac{\phi_{a}{ }^{n+1}}{(n+1) !}-\frac{\phi_{b}{ }^{n+1}}{(n+1) !}\right)
$$


La energía potencial gravitatoria neta $E$ entre $a$ y $b$ estaría dada por la sumatoria de todas las energías potenciales gravitatorias de enésimo nivel cuando $n \rightarrow \infty$, es decir:

$$
E=\sum_{n=0}^{n \rightarrow \infty}(-1)^{n+1} \frac{G M^{2}}{\lambda}\left(\frac{\phi_{a}{ }^{n+1}}{(n+1) !}-\frac{\phi_{b}{ }^{n+1}}{(n+1) !}\right)
$$

Sumando y restando 1 dentro del paréntesis obtenemos:

$$
E=-\frac{G M^{2}}{\lambda}\left(e^{-\frac{\lambda}{b}}-e^{-\frac{\lambda}{a}}\right)
$$

Ahora bien, esta ecuación nos da la diferencia de energía potencial gravitatoria entre dos puntos situados a distancias $a$ y $b$ del centro de $M$. Podemos asignar un valor a un punto situado a una distancia $r$ de la masa, para lo cual es necesario elegir un punto de referencia arbitrario al que se le asigna el potencial 0. Para satisfacer esta condición, dicho punto debe hallarse a una distancia infinita. Si asignamos el punto $r$ en $a$ y el infinito en $b$ obtendremos la energía potencial gravitatoria en $r$ :

$$
E=-\frac{G M^{2}}{\lambda}\left(1-e^{-\frac{\lambda}{r}}\right)
$$

O bien:

$$
E=\frac{G M^{2}}{\lambda}\left(e^{-\frac{\lambda}{r}}-1\right)
$$

Esta energía es siempre negativa. Cuando $r \rightarrow 0$, entonces:

$$
E=-\frac{G M^{2}}{\lambda}
$$

que es una cantidad finita. Anteriormente definimos $\lambda$ como una longitud vinculada a $M$. Podemos elegir $\lambda$ de manera que $M / \lambda=k$, siendo $k$ una constante que define el vínculo entre $M \quad$ y $\lambda$, luego $G M / \lambda$ será también otra constante equivalente al cuadrado de una velocidad. Ahora bien, la única velocidad constante en la naturaleza es la velocidad de la luz, de modo que podemos asumir que GM/ $\lambda=c^{2}$, de donde $\lambda=G M / c^{2}$. Reemplazando este valor en la ecuación 2.12 tenemos:

$$
E=-M c^{2}
$$


En el caso del electrón que analizamos en una anterior publicación ya referida [7], la energía electrostática cuando $r \rightarrow 0$ está dada por $E=m_{e} c^{2}$.

De modo análogo la energía gravitatoria de $M$ cuando $r \rightarrow 0$ estaría dada por $E=-M c^{2}$.

Es decir, la energía gravitatoria de una masa $M$ cuando $\quad r \rightarrow 0$ es su energía intrínseca con signo negativo.

Se concluye entonces que la energía gravitatoria de un agujero negro de masa $M$ es una magnitud finita en el límite newtoniano modificado y consecuentemente en la relatividad general modificada, a diferencia de los modelos newtoniano y relativista que dan un valor infinito.

Este importante resultado excluye una magnitud física infinita para la energía gravitatoria al que conduce la relatividad general.

Cabe señalar que el método de la renormalización en la electrodinámica cuántica no ha podido aplicarse a la gravedad en la que no se ha logrado eliminar la divergencia infinita, a diferencia del modelo propuesto por nosotros que sí ha conseguido eliminar la divergencia infinita en la gravedad.

La energía gravitatoria de un agujero negro tendría un límite natural. Este resultado nos conduce además a la posibilidad de establecer un origen para las fuerzas fundamentales de la naturaleza, incluyendo la gravedad, como gradientes diferenciados de un mismo origen que es la energía intrínseca $E=M c^{2}$.

Es importante indicar que la energía gravitatoria en la relatividad general se da en función de la energía relativista intrínseca de la masa, de manera que la aceleración aparente que se deriva a partir de ella no entra en contradicción con el postulado de la relatividad general de considerar la gravedad como una geometría del espaciotiempo y no como una fuerza.
La energía gravitatoria es $-n M c$, donde $0 \leq n \leq 1$. Cuando $n=1$, se obtiene la energía máxima negativa $-M c^{2}$

Esta energía sería la misma en la mecánica newtoniana y en la relatividad general. Su derivada respecto a la distancia nos da la fuerza gravitatoria newtoniana o la fuerza o aceleración aparentes de la relatividad general.

Es precisamente la energía intrínseca de la masa donde se integraría la relatividad general a las demás fuerzas fundamentales de la naturaleza.

Reemplazando el valor $\lambda=G M / c^{2}$ en la ecuación 2.11 tendremos:

$$
E=M c^{2}\left(e^{-\frac{G M}{c^{2} r}}-1\right)
$$

Ecuación similar a la energía del electrón modificada, con signo negativo:

$$
E=m_{e} c^{2}\left(1-e^{-\frac{k e^{-^{2}}}{m_{e} c^{2} r}}\right)
$$

donde $k$ es la constante coulombiana.

Expandiendo el exponencial:

$$
E=-\frac{G M^{2}}{r}+\frac{G^{2} M^{4}}{2 ! r^{2}}-\cdots
$$

Despreciando el segundo término en adelante por tratarse de distancias relativamente grandes tendremos:

$$
E \approx \frac{G M^{2}}{r}
$$

Que es la ecuación de la energía potencial gravitatoria en el límite newtoniano como caso particular. Dividiendo la ecuación 2.17 por $M$ obtendremos el potencial newtoniano gravitatorio $V$ de $M$ en $r$ :

$$
V \approx-\frac{G M}{r}
$$


Dividiendo la ecuación 2.14 por $M$ obtendremos el potencial newtoniano gravitatorio modificado $V$ de $M$ en $r$ :

$$
V=c^{2}\left(e^{-\frac{G M}{c^{2} r}}-1\right)
$$

La gráfica de la figura 6, para $G M / c^{2}=1$, muestra los potenciales gravitatorios newtoniano clásico en azul y modificado en rojo, en función de la distancia al centro del agujero negro:

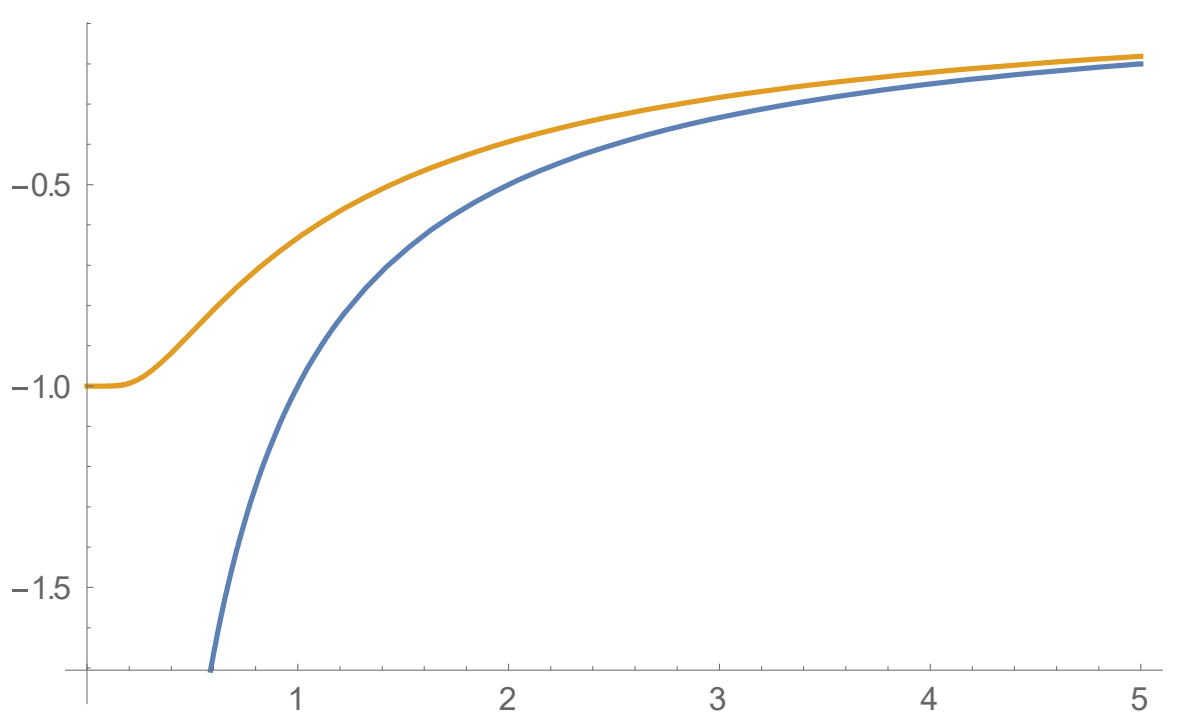

Figura $N^{\circ}$ 6. Gráfica comparativa entre los potenciales gravitatorio newtoniano clásico en azul y modificado en rojo en función de la distancia al centro del agujero negro.

Derivando las ecuaciones 2.18 y 2.19 respecto a $r$ obtendremos la intensidad $g$ del campo gravitatorio newtoniano clásico y modificado:

$$
\begin{aligned}
& \boldsymbol{g} \approx-\frac{G M}{r^{2}} \boldsymbol{r} \\
& \boldsymbol{g}=-\frac{G M}{r^{2}} e^{-\frac{G M}{c^{2} r}} \boldsymbol{r}
\end{aligned}
$$


Cuya gráfica es:

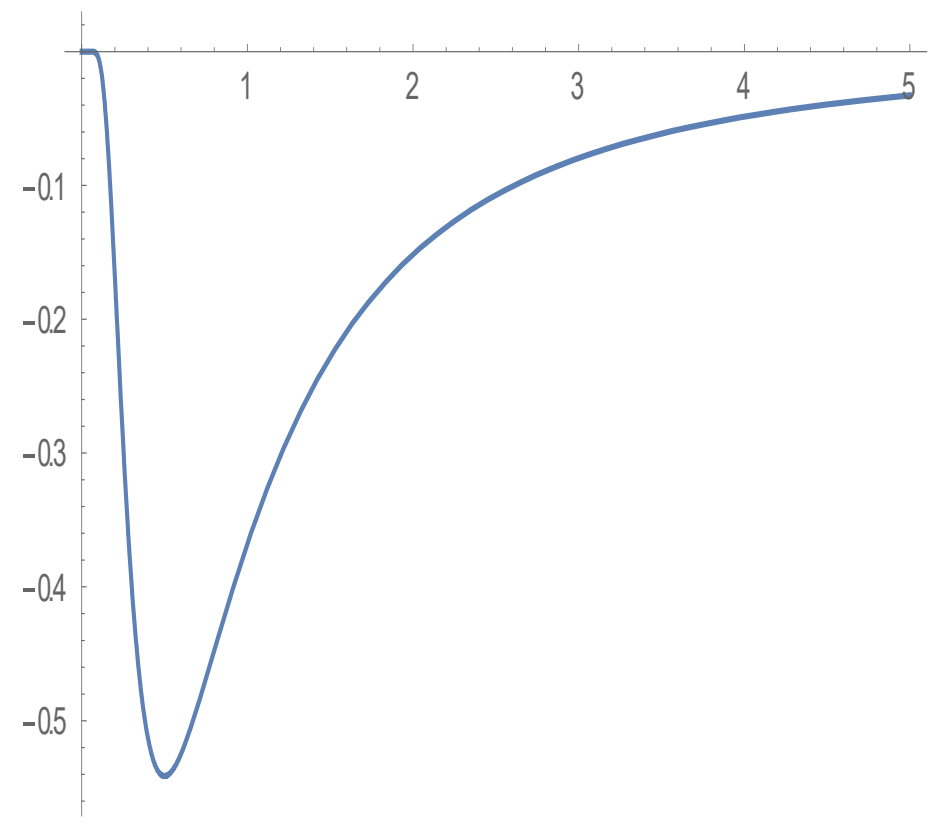

Figura No 7. Gráfica de la intensidad del campo gravitatorio modificado

\section{Métrica de Schwarzschild modificada.}

Consideremos una masa estática $M$ de simetría esférica. La métrica en coordenadas $(r, t)$ para una masa sin rotación y en ausencia de campo gravitatorio respecto a sí misma en el cascarón esférico de radio $r$ es la métrica de Minkowski:

$$
d s^{2}=c^{2} d t^{2}-d r^{2}
$$

Derivando con respecto al tiempo propio $\tau$ tendremos:

$$
c^{2}=c^{2} \dot{t}^{2}-\dot{r}^{2}
$$

En presencia de un campo gravitacional tendremos:

$$
c^{2}=A c^{2} \dot{t}^{2}-B \dot{r}^{2}
$$

donde A y B son coeficientes por determinar que definen la curvatura espaciotemporal. Se demuestra que $A c^{2} \dot{t}$ es una constante igual a la energía relativista por unidad de masa, es decir, $A c^{2} \dot{t}=E / M$, de donde $\dot{t}^{2}=E^{2} / A^{2} M^{2} c^{4}$. Reemplazando este valor en la ecuación 3.3 obtenemos: 


$$
c^{2}=\frac{E^{2}}{A M^{2} c^{2}}-B \dot{r}^{2}
$$

Resolviendo:

$$
E^{2}=A M^{2} c^{4}+A B M^{2} c^{2} \dot{r}^{2}
$$

De esta ecuación vamos a obtener la energía potencial gravitatoria de $M$. Restando $M^{2} c^{4}$ y dividiendo por $2 M c^{2}$ tenemos:

$$
\frac{E^{2}-M^{2} c^{4}}{2 M c^{2}}=E_{\varphi}=\frac{A M c^{2}}{2}+\frac{A B M \dot{r}^{2}}{2}-\frac{M c^{2}}{2}
$$

siendo $\mathrm{E} \varphi$ una nueva energía. Ahora bien, podemos determinar valores para $A$ y $B$ de manera que $E \varphi$ represente la energía potencial gravitatoria de $M$ respecto a sí misma, es decir:

$$
E_{\varphi}=\frac{A M c^{2}}{2}+\frac{A B M \dot{r}^{2}}{2}-\frac{M c^{2}}{2} \approx-\frac{G M^{2}}{r}+\frac{M \dot{r}^{2}}{2}
$$

según la ecuación 3.6 más la energía cinética de $M$. Para satisfacer la ecuación anterior $A B M \dot{r}^{2} / 2$ debe ser igual a la energía cinética de $M$, es decir, $A B M \dot{r}^{2} / 2=M \dot{r}^{2} / 2$, por tanto $A B=1$, de donde $B=1 / A$. Como $M$ es una masa estática, en reposo sin rotación, $\dot{r}=0$ y $M \dot{r}^{2} / 2=0$, luego:

$$
E_{\varphi}=\frac{A M c^{2}}{2}-\frac{M c^{2}}{2} \approx-\frac{G M^{2}}{r}
$$

de donde:

$$
A \approx 1-\frac{2 G M}{c^{2} r}
$$

Sustituyendo la ecuación 3.9 en la ecuación 3.3 tendremos la métrica de Schwarzschild para una masa estática de simetría esférica en coordenadas $(t, r)$ :

$$
c^{2}=\left(1-\frac{2 G M}{c^{2} r}\right) c^{2} \dot{t}^{2}-\left(1-\frac{2 G M}{c^{2} r}\right)^{-1} \dot{r}^{2}
$$


Schwarzschild obtuvo los coeficientes $A$ y $B$ por otro método basado en el análisis tensorial para un tensor de Ricci $R_{u v}=0$ y en el potencial newtoniano $-G M / r$ como una solución exacta a las ecuaciones de campo de Einstein.

Si sustituimos $-G M^{2} / r$ por la ecuación 2.14 tendremos:

$$
E_{\varphi}=\frac{A M c^{2}}{2}-\frac{M c^{2}}{2}=M c^{2}\left(e^{-\frac{G M}{c^{2} r}}-1\right)
$$

de donde:

$$
A=2 e^{-\frac{G M}{c^{2} r}}-1
$$

Reemplazando este valor en la ecuación 3.3 tendremos la métrica de Schwarzschild modificada:

$$
c^{2}=\left(2 e^{-\frac{G M}{c^{2} r}}-1\right) c^{2} \dot{t}^{2}-\left(2 e^{-\frac{G M}{c^{2} r}}-1\right)^{-1} \dot{r}^{2}
$$

Si en la métrica de Schwarzschild sustituimos el potencial newtoniano por el potencial newtoniano modificado de la ecuación 2.19 obtendremos el mismo resultado. En las métricas para agujeros negros en rotación y con carga eléctrica, así como todas las métricas basadas en el potencial newtoniano, debe reemplazarse este potencial por el potencial newtoniano modificado.

\section{Geodésicas.}

El elemento de línea viene dado por:

$$
d s^{2}=-A c^{2} t^{2}+B \dot{r}^{2}+r^{2} d \theta^{2}+r^{2} \operatorname{sen} \theta d \phi^{2}\left(x^{0}, x^{1}, x^{2}, x^{3}\right)=(c t, r, \theta, \phi)
$$

donde $A$ y $B$ son los coeficientes de la métrica modificada de Schwarzschild. Definamos $A^{\prime} y B^{\prime}$ como las derivadas de $A$ y $B$ respectivamente respecto a $r$, siendo sus valores:

$$
\begin{gathered}
A^{\prime}=\frac{2 G M}{c^{2} r^{2}} e^{-\frac{G M}{c^{2} r}} \\
B^{\prime}=-\frac{2 G M}{c^{2} r^{2}}\left(2 e^{-\frac{G M}{c^{2} r}}-1\right)^{-2} e^{-\frac{G M}{c^{2} r}}
\end{gathered}
$$


El tensor métrico es:

$$
g_{u v}=\left[\begin{array}{cccc}
-A & 0 & 0 & 0 \\
0 & B & 0 & 0 \\
0 & 0 & r^{2} & 0 \\
0 & 0 & 0 & r^{2} \operatorname{sen}^{2} \theta
\end{array}\right]
$$

Los símbolos de Christoffel son:

$\Gamma_{p q}^{0}=\left[\begin{array}{cccc}0 & \frac{A^{\prime}}{2 A} & 0 & 0 \\ \frac{A^{\prime}}{2 A} & 0 & 0 & 0 \\ 0 & 0 & 0 & 0 \\ 0 & 0 & 0 & 0\end{array}\right] \quad \Gamma_{p q}^{1}=\left[\begin{array}{cccc}\frac{A^{\prime}}{2 B} & 0 & 0 & 0 \\ 0 & \frac{B^{\prime}}{2 B} & 0 & 0 \\ 0 & 0 & \frac{-r}{B} & 0 \\ 0 & 0 & 0 & \frac{-r \operatorname{sen}^{2} \theta}{B}\end{array}\right]$

$$
\Gamma_{p q}^{2}=\left[\begin{array}{cccc}
0 & 0 & 0 & 0 \\
0 & 0 & \frac{1}{r} & 0 \\
0 & \frac{1}{r} & 0 & 0 \\
0 & 0 & 0 & -\operatorname{sen} \theta \cos \theta
\end{array}\right] \Gamma_{p q}^{3}=\left[\begin{array}{cccc}
0 & 0 & 0 & 0 \\
0 & 0 & 0 & \frac{1}{r} \\
0 & 0 & 0 & \cot \theta \\
0 & \frac{1}{r} & \cot \theta & 0
\end{array}\right]
$$

La ecuación para encontrar las geodésicas es:

$$
\ddot{x}^{r}+\Gamma_{p q}^{r} \dot{x}^{p} \dot{x}^{q}=0
$$


En el presente caso:

$$
\begin{gathered}
\ddot{t}+\frac{A^{\prime}}{A} \dot{t} \dot{r}=0 \\
\ddot{r}+\frac{A^{\prime}}{2 B} \dot{t}^{2}+\frac{B^{\prime}}{2 B}+\dot{r}^{2}-\frac{r}{B}\left(\dot{\phi}^{2}+\operatorname{sen}^{2} \theta\right) \dot{\phi}^{2} \\
\ddot{\theta}+\frac{2}{r} \dot{r} \dot{\theta}-\operatorname{sen} \theta \cos \theta \dot{\phi}^{2}=0 \\
\ddot{\phi}+\frac{2}{r} \dot{r} \dot{\phi}+2 \cot \theta \dot{\phi} \dot{\theta}=0
\end{gathered}
$$

\section{Ecuaciones de movimiento y precesión de la órbita de los planetas.}

La métrica de Minkowski con simetría esférica en coordenadas $(t, r, \theta, \phi)$ y en ausencia de campo es:

$$
d s^{2}=c^{2} d t^{2}-d r^{2}-r^{2} d \theta^{2}-r^{2} \operatorname{sen} \theta d \phi^{2}
$$

En presencia de campo y asumiendo que la métrica no depende del tiempo y adoptando el gauge estándar, en donde la métrica no es afectada en los términos del diferencial del ángulo sólido, tendremos la métrica de Schwarzschild modificada:

$$
d s^{2}=\left(2 e^{-\frac{G M}{c^{2} r}}-1\right) c^{2} d t^{2}-\left(2 e^{-\frac{G M}{c^{2} r}}-1\right)^{-1} d r^{2}-r^{2} d \theta^{2}-r^{2} \sin \theta d \phi^{2}
$$

El lagrangiano $L$ de esta ecuación es:

$$
\mathcal{L}=c=\left[\left(2 e^{-\frac{G M}{c^{2} r}}-1\right) c^{2} \dot{t}^{2}-\left(2 e^{-\frac{G M}{c^{2} r}}-1\right)^{-1} \dot{r}^{2}-r^{2} \dot{\theta}^{2}-r^{2} \operatorname{sen} \theta \dot{\phi}^{2}\right]^{\frac{1}{2}}
$$

En la ecuación de movimiento para $t$, para una masa $\mathrm{m}$ que gira alrededor de una masa $M$, como $\partial \mathcal{L} / \partial t=0$, entonces se tiene que $d(\partial \mathcal{L} / \partial \dot{t}) / d \tau=0$. Es decir, $\partial \mathcal{L} / \partial \dot{t}=c t e$. Dicha constante es la energía relativista por unidad de masa $\mathrm{m}$ :

$$
\left(2 e^{-\frac{G M}{c^{2} r}}-1\right) c^{2} \dot{t}=\frac{E}{m}
$$


Cuando $r \rightarrow \infty$ se debe tener que $\left(2 e^{-\frac{G M}{c^{2} r}}-1\right) \rightarrow 1$, de manera que nos encontramos en la métrica de Minkowski con $E=m \gamma c^{2}$, siendo $\dot{t}=r=1 / \sqrt{1-v^{2} / c^{2}}$, donde $\mathrm{v}$ es la velocidad de $m$ respecto a un observador. En la ecuación de movimiento para $\phi$, como $\partial L \partial \phi=0$ entonces se tiene que $d(\partial \mathcal{L} / \partial \dot{\phi}) / d \tau=0$. Es decir $\partial \mathcal{L} / \partial \dot{\phi}=$ cte. Dicha constante es el momento angular relativista $l$ por unidad de masa:

$$
r^{2} \operatorname{sen}^{2} \theta \dot{\phi}=\frac{l}{m}
$$

Se puede demostrar que el movimiento entre dos cuerpos está en un plano. Para $\theta=\pi / 2$, en la ecuación 5.3 tenemos:

$$
c^{2}=\left(2 e^{-\frac{G M}{c^{2} r}}-1\right) c^{2} \dot{t}^{2}-\left(2 e^{-\frac{G M}{c^{2} r}}-1\right)^{-1} \dot{r}^{2}-r^{2} \dot{\phi}^{2}
$$

Reemplazando las ecuaciones 5.4 y 5.5 en la ecuación 5.6 tendremos:

$$
c^{2}=\left(2 e^{-\frac{G M}{c^{2} r}}-1\right)^{-1} \frac{E^{2}}{m^{2} c^{2}}-\left(2 e^{-\frac{G M}{c^{2} r}}-1\right)^{-1} \dot{r}^{2}-\frac{l^{2}}{m^{2} r^{2}}
$$

Despejando $E^{2}$, restando $m^{2} c^{4}$ y dividiendo por $2 m c^{2}$ en la ecuación 5.7 tendremos una nueva energía $E \varphi$ que es la energía potencial gravitatoria de $m$ respecto a $M$ en $r$ :

$$
E_{\varphi}=m c^{2} e^{-\frac{G M}{c^{2} r}}-m c^{2}+\frac{m \dot{r}^{2}}{2}+\frac{l^{2}}{m r^{2}} e^{-\frac{G M}{c^{2} r}}-\frac{l^{2}}{2 m r^{2}}
$$

Derivando respecto a $\tau$ tenemos:

$$
0=\frac{G M m}{r^{2}} e^{-\frac{G M}{c^{2} r}} \dot{r}+m \dot{r} \ddot{r}+\frac{G M l^{2}}{m c^{2} r^{4}} e^{-\frac{G M}{c^{2} r}} \dot{r}-\frac{2 l^{2}}{m r^{3}} r+\frac{l^{2}}{m^{2} r^{3}}
$$

Despejando $r ":$

$$
\ddot{r}=-\frac{G M}{r^{2}} e^{-\frac{G M}{c^{2} r}}-\frac{G M l^{2}}{m^{2} c^{2} r^{4}} e^{-\frac{G M}{c^{2} r}}+\frac{2 l^{2}}{m^{2} r^{3}} e^{-\frac{G M}{c^{2} r}}-\frac{l^{2}}{m^{2} r^{3}}
$$

Por otro lado la expresión para la aceleración radial $a_{r}$ viene dada por $a_{r}=\ddot{r}-r \dot{\theta}^{2}-r \operatorname{sen}^{2} \theta \dot{\phi}^{2}$ Si consideramos $\theta=\pi / 2$ tendremos:

$$
a_{r}=\ddot{r}-r \dot{\phi}^{2}
$$


Teniendo en cuenta que $l=m r^{2} \dot{\phi}$ y la ecuación 5.11 tenemos:

$$
a_{r}=-\frac{G M}{r^{2}} e^{-\frac{G M}{c^{2} r}}-\frac{G M l^{2}}{m^{2} c^{2} r^{4}} e^{-\frac{G M}{c^{2} r}}+\frac{2 l^{2}}{m^{2} m^{3}} e^{-\frac{G M}{c^{2} r}}-\frac{2 l^{2}}{m^{2} r^{3}}
$$

Esta es la ecuación exacta de la aceleración radial de $m$ respecto a $M$ Expandiendo el exponencial en la ecuación tenemos:

$$
a_{r}=-\frac{G M}{r^{2}}+\frac{G^{2} M^{2}}{c^{2} r^{2}}-\cdots-\frac{G M l^{2}}{m^{2} c^{2} r^{4}}+\frac{G^{2} M^{2} l^{2}}{m^{2} c^{2} r^{5}}-\cdots+\frac{2 l^{2}}{m^{2} r^{2}}-\frac{2 G M l^{2}}{m^{2} c^{2} r^{4}}+\frac{G^{2} M^{2} l^{2}}{m^{2} c^{4} r^{5}}-\frac{2 l^{2}}{m^{2} r^{2}}
$$

Despreciando en las series los términos para distancias relativamente grandes tenemos:

$$
a_{r} \approx-\frac{G M}{r^{2}}\left(1+\frac{3 l^{2}}{m^{2} c^{2} r^{2}}\right)+\frac{G^{2} M^{2}}{c^{2} r^{3}}\left(1+\frac{2 l^{2}}{m^{2} c^{2} r^{2}}\right)
$$

El primer sumando de la ecuación corresponde a la ecuación del movimiento en la métrica de Schwarzschild con una modificación relativista que explica satisfactoriamente la precesión del perihelio de Mercurio de 43 segundos de arco por siglo. Se trata de una aproximación al límite relativista.

El segundo sumando corresponde a la modificación introducida por nosotros, equivalente aproximadamente a un desplazamiento en sentido contrario del perihelio por el orden de $10^{-8}$ segundos de arco por siglo, magnitud difícil de detectar, considerando que el efecto cuántico de las partículas virtuales de antimateria se ponen de manifiesto de modo significativo a distancias muy cortas.

Con el descubrimiento de nuevos exoplanetas o cuerpos masivos girando alrededor de estrellas muy densas, tal vez en el futuro puedan detectarse estos desplazamientos.
Expandiendo el exponencial de la ecuación en la métrica de Schwarzschild modificada y despreciando los términos de la serie a partir del tercer término por tratarse de distancias relativamente grandes obtendremos la métrica de Schwarzschild en el límite relativista, como caso particular.

Cuando $r \rightarrow \infty$ obtendremos la métrica de Minkowski, coincidiendo con la métrica de Schwarzschild y la modificada a una distancia situada en el infinito libre de un campo gravitatorio. Cuando $r \rightarrow 0$, obtenemos nuevamente una métrica de Minkowski, coincidiendo con la ausencia de campo gravitatorio en el centro del agujero negro, con los signos cambiados.

La gráfica de la figura 8 muestra los coeficientes $A$ en azul y $B$ en rojo de la métrica de Schwarzschild modificada respecto a la distancia $r$ para $G M / c^{2}=1$. 


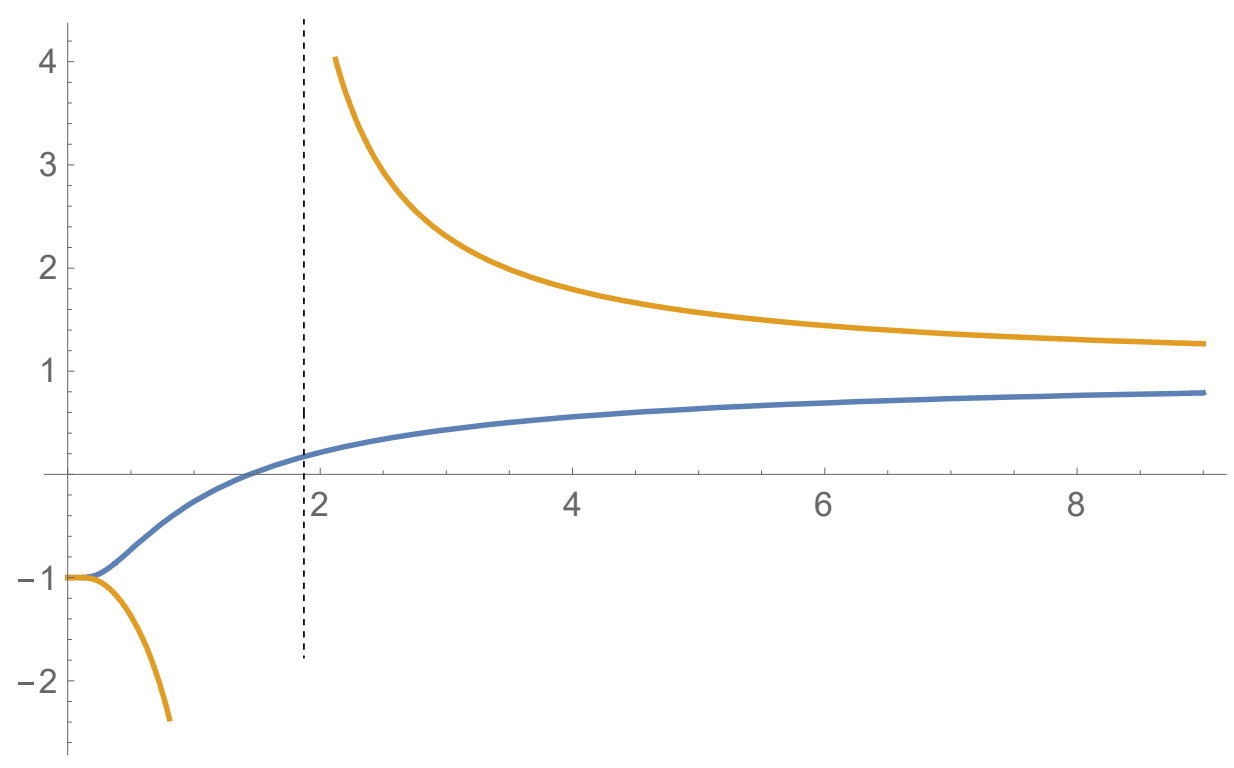

Figura No 8. Gráfica de los coeficientes $A$ y $B$ en la métrica modificada de Schwarzschild.

Cuando $A=0$ obtendremos un nuevo radio $r \varphi$ para el horizonte de eventos, cuyo valor está dado por:

$$
r_{\varphi}=\frac{G M}{c^{2} \ln [2]}
$$

magnitud menor que el radio de Schwarzschild para el horizonte de eventos dado por $r_{s}=2 G M / c^{2}$ La gráfica muestra una simetría central en diferentes escalas para los coeficientes $A$ y $B$, una elegancia matemática que no se presenta en la métrica de Schwarzschild [8]. A cada punto $\mathrm{r}_{1}$ comprendido entre 0 y $r_{\varphi}$, le corresponde otro punto $\mathrm{r}$ comprendido entre $r_{\varphi}$ e infinito, de manera que los coeficientes A y B del primer punto son los mismos coeficientes del segundo punto con el signo opuesto. De los coeficientes $A$ y $B$ se deduce que:

$$
\begin{gathered}
r_{2}=-\frac{G M}{c^{2} r_{1} \ln \left[1-e^{-\frac{G M}{c^{2} r_{1}}}\right]} \\
0 \leq r_{1} \leq \frac{G M}{c^{2} \ln [2]}
\end{gathered}
$$


Cuya gráfica para $G M / c^{2}=1$ está representada por la figura 9:

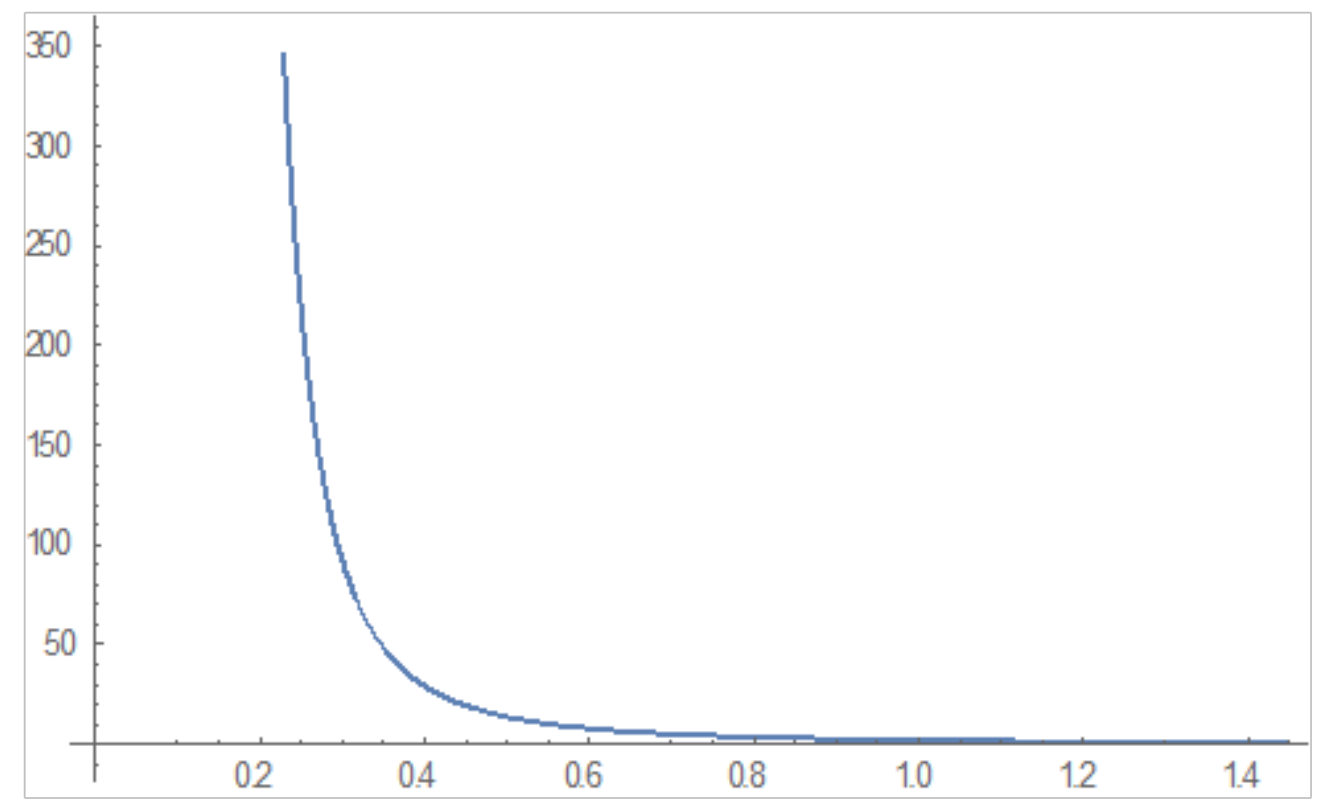

Figura No 9. Gráfica de los coeficientes $A$ y $\quad B$ entre el centro del agujero negro y el horizonte de eventos.

\section{Agujeros negros de antimateria.}

Esta simetría central muestra claramente una simetría CPT (carga, paridad y tiempo), en la que el espacio y el tiempo se distorsionan e invierten dando origen a un sistema de coordenadas que corresponden a un objeto de antimateria visto por un observador que se encuentra más allá del horizonte de eventos, lo que lleva a la conclusión de que los agujeros negros son de antimateria y en consecuencia, al tratarse de una carga gravitatoria imaginaria $-M i$ como señalamos anteriormente, generará una repulsión gravitatoria a toda la materia que se encuentre a una distancia mayor al radio $\mathrm{r} \varphi$ que determina el horizonte de eventos.

La conclusión de que los agujeros negros son de antimateria y en consecuencia repelen la materia que se encuentra más allá del horizonte de eventos cambia por completo todo lo investigado sobre agujeros negros hasta la fecha.

La masa $M$ que dio origen al agujero negro mantiene la atracción gravitatoria sobre ella misma durante el colapso gravitatorio, ya que solo repele a masas separadas de ella por una distancia mayor que $r \varphi$ y atrae a masas que se encuentren a distancias menores que $r \varphi$, puesto que dos masas de antimateria se atraen entre sí. Por otro lado, desde la perspectiva de un observador que se encuentre en la masa $M$ no se produce ningún cambio en la masa, a diferencia de las masas que se encuentren en un radio mayor que $r \varphi$, que se transformarían en antimateria para este observador.

La intensidad del campo gravitatorio para $r \geq r_{\varphi}$ y $\mathrm{GM} / c^{2}=1$ es la gráfica de la figura 7 invertida.

Cabe señalar que como consecuencia del principio de simetría CPT analizado con mayor detalle en otra publicación [9], el tiempo de las partículas virtuales de antimateria transcurre del pasado hacia el futuro, pero en sentido opuesto al de la materia, de manera que estas partículas virtuales neutralizan el retardo temporal relativista en la métrica de Schwarzschild. 
Este nuevo resultado difiere sustancialmente de la relatividad general que se basa en el potencial newtoniano y no en el potencial modificado por las partículas virtuales de antimateria.

Con la nueva métrica eliminamos los infinitos de la singularidad espaciotemporal basada en la relatividad cuando $r \rightarrow 0$. Los rayos de luz y cualquier señal electromagnética quedan atrapados dentro del horizonte de eventos.
A medida que una estrella masiva colapsa, su tiempo, visto por un observador externo, se va ralentizando hasta detenerse en el horizonte de eventos, a partir del cual el tiempo retrocede lentamente hasta transcurrir normalmente hacia el pasado hasta un punto donde la variación del tiempo propio coincide con la del observador en el centro del agujero negro, proceso en el cual la entropía se invierte. La gráfica de la figura 10 muestra los conos de luz de este proceso.

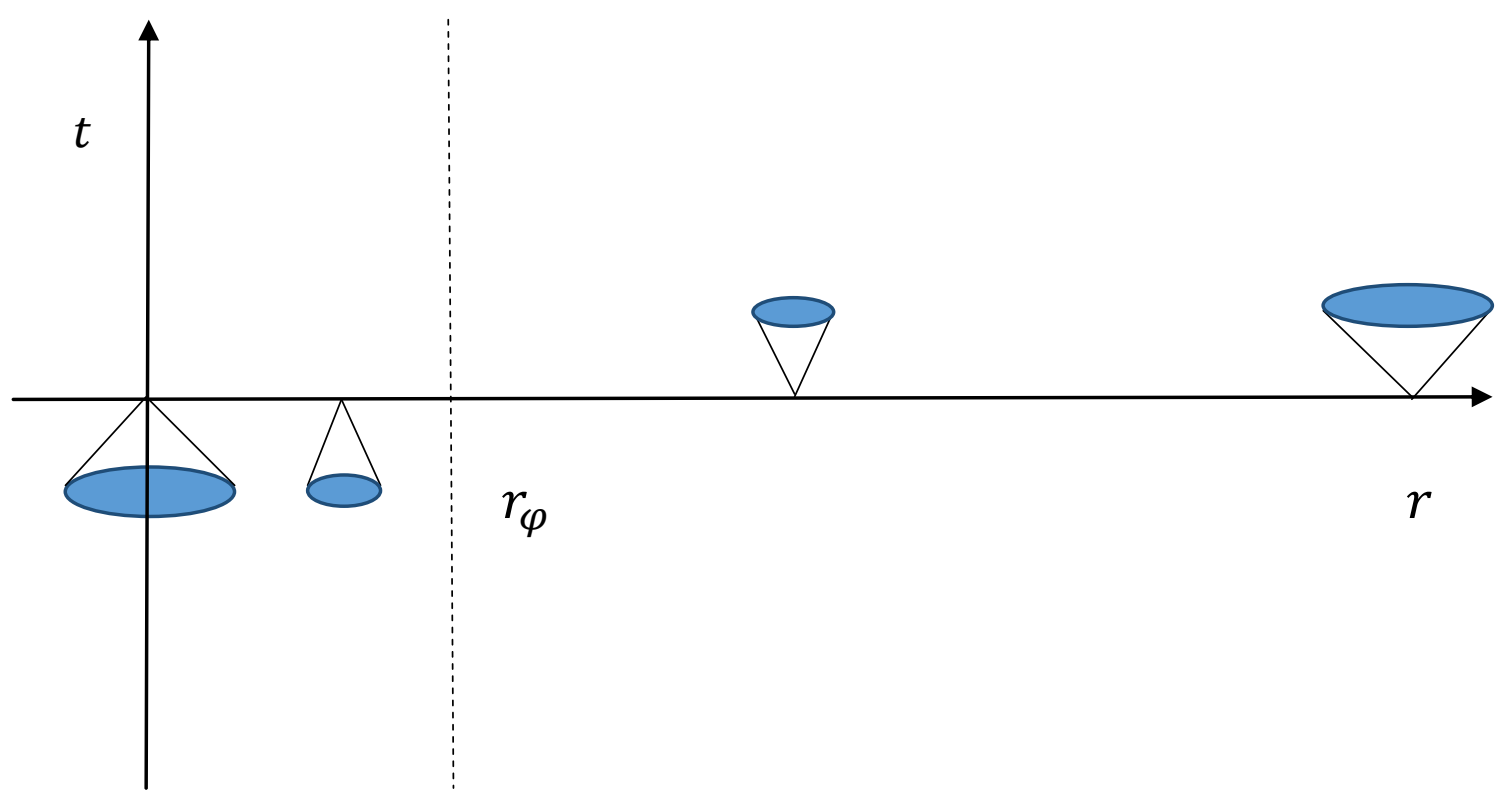

Figura $\mathrm{N}^{\circ}$ 10. Representación gráfica de los conos de luz en función de la distancia al centro del agujero negro.

Lo mismo ocurre para un observador situado dentro del horizonte de eventos para el cual el tiempo transcurre normalmente hacia el futuro. Para un observador situado en el centro del agujero negro, el tiempo se ralentiza a medida que se aleja de ese centro, hasta que se detiene en el horizonte de eventos.

A partir de allí comienza a transcurrir lentamente hacia el pasado a medida que se aleja más allá del horizonte, hasta transcurrir normalmente hacia el pasado donde la variación del tiempo propio coincide con la del observador a una distancia infinita del centro del agujero negro, en una región libre de influencias gravitatorias, es decir, un espacio minkowskiano como el que existe en el centro del agujero negro. Este proceso está representado también por la figura 10 pero con los conos invertidos. La entropía se invierte para objetos situados más allá del horizonte de eventos.

Si bien ningún observador externo o interno puede ver lo que ocurre más allá del horizonte de eventos, las matemáticas nos permiten deducir el comportamiento de estos sucesos. Sin embargo, 
el efecto antigravitatorio de los agujeros negros es algo que se puede observar y comprobar experimentalmente.

\section{Materia oscura.}

Esto podría explicar el comportamiento observado en la velocidad de rotación en los cinturones de estrellas que giran alrededor del centro de su galaxia, atribuido a la presencia de materia oscura. Al igual que las partículas virtuales de antimateria que ralentizan la rotación de los planetas alrededor de una estrella, así también un agujero negro de antimateria ralentizaría la rotación de las estrellas alrededor del centro de la galaxia, efecto que disminuiría con la distancia, de manera que las estrellas más cercanas al centro girarían con menor velocidad que las más lejanas, aproximando sus velocidades de rotación.

La atracción gravitatoria de las estrellas en las galaxias neutralizaría parcialmente la repulsión gravitatoria del agujero negro manteniendo sus trayectorias circulares alrededor del mismo.

\section{CONCLUSIONES}

La hipótesis de los subniveles fractales del vacío cuántico y la interacción de sus respectivas partículas virtuales de materia y antimateria explicaría satisfactoriamente el comportamiento de las partículas reales elementales consideradas como partículas puntuales según el modelo estándar, sin tener que recurrir a la renormalización, eliminando la singularidad espaciotemporal en el centro de los agujeros negros y los infinitos en la relatividad general, donde la masa se transforma en antimateria en el interior del horizonte de eventos.

\section{REFERENCIAS}

[1] Álvarez Vita, Enrique. Universos fractales de materia y antimateria y el neutrovacío. Revista Ciencia y Desarrollo, UAP, Vol. 18, Num. 1, p. 71, 2015.[Internet]. Disponible: revistas.uap.edu.pe/ ojs/index.php/CYD/article/view/1088
[2] Álvarez Vita, Enrique. Apantallamiento gravitatorio y agujeros negros. Revista Ciencia y Desarrollo, UAP, Vol. 18, Num. 2, p. 79 2015. [Internet].Disponible: revistas.uap.edu.pe/ojs/index. php/CYD/article/view/1082

[3] Álvarez Vita, Enrique. Apantallamiento gravitatorio y agujeros negros. Revista Ciencia y Desarrollo, UAP, Vol. 18, Num. 2, p. 80 2015.[Internet].Disponible: revistas.uap.edu.pe/ojs/index. php/CYD/article/view/1082

[4] Álvarez Vita, Enrique. Universos fractales de materia y antimateria y el neutrovacío. Revista Ciencia y Desarrollo, UAP, Vol. 18, Num. 1, p. 7980, 2015.[Internet].Disponible: revistas.uap.edu. pe/ojs/index.php/CYD/article/view/1088

[5] Álvarez Vita, Enrique. Universos fractales de materia y antimateria y el neutrovacío. Revista Ciencia y Desarrollo, UAP, Vol. 18, Num. 1, p. 81, 2015.[Internet].Disponible: revistas.uap.edu.pe/ ojs/index.php/CYD/article/view/1088

[6] Álvarez Vita, Enrique. Universos fractales de materia y antimateria y el neutrovacío. Revista Ciencia y Desarrollo,UAP, Vol. 18, Num. 1, p. 82, 2015.[Internet]. Disponible:revistas.uap.edu.pe/ ojs/index.php/CYD/article/view/1088

[7] Álvarez Vita, Enrique. Universos fractales de materia y antimateria y el neutrovacío. Revista Ciencia y Desarrollo, UAP, Vol. 18, Num. 1, p.7275, 2015.[Internet]. Disponible: revistas.uap.edu. pe/ojs/index.php/CYD/article/view/1088

[8] Álvarez Vita, Enrique. Universos fractales de materia y antimateria y el neutrovacío. Revista Ciencia y Desarrollo, UAP, Vol. 18, Num. 1, p.78, 2015.[Internet]. Disponible: revistas.uap.edu.pe/ ojs/index.php/CYD/article/view/1088

[9] Álvarez Vita, Enrique. Universos fractales de materia y antimateria y el neutrovacío. Revista Ciencia y Desarrollo, UAP, Vol. 18, Num. 1, p.7677, 2015.[Internet]. Disponible: revistas.uap.edu. pe/ojs/index.php/CYD/article/view/1088 


\section{BIBLIOGRAFÍA.}

Alexandrov, A.d. Komologorov, A.n. Laurentiev, M.a. y otros. La matemática: su contenido, métodos y significado. Tomo. I, II y III. Alianza Editorial, S.A. Madrid, 1981.

Álvarez Vita, Enrique. La belleza como guía de la ciencia. Revista Tradición, Año XI, No 11, Universidad Ricardo Palma, URP. Lima, 2011.

Álvarez Vita, Enrique. Universos de materia y antimateria. Revista Tradición, Año XIII, N 13 , Universidad Ricardo Palma. Lima, 2013.

Álvarez Vita, Enrique. Un esbozo sobre la unificación de las fuerzas fundamentales de la naturaleza y sus implicaciones filosóficas. Revista Evohé, Año III, Nº 3, Revista Villarrealina de Filosofía. Lima, 2014.

Álvarez Vita, Enrique. Universos fractales de materia y antimateria y el neutrovacío. Revista Ciencia y Desarrollo, volumen 18, número 1, enero - junio 2015. Universidad Alas Peruanas. Lima, 2015.

Álvarez Vita, Enrique. Apantallamiento gravitatorio y agujeros negros. Revista Ciencia y Desarrollo, volumen 18 , número 2 , julio - diciembre 2015. Universidad Alas Peruanas. Lima, 2015.

Álvarez Vita, Enrique. La gravedad modificada. Revista Tradición, Año XVI, N 16 , Universidad Ricardo Palma, URP. Lima, 2016.

Calcina, Esly Abner. Agujeros negros. Informe de Tópicos de Investigación II, Facultad de Ciencias, Universidad Nacional de Ingeniería. Lima, 2014.

Calcina, Esly Abner. Métrica con simetría radial. Lima, 2015.

De la Torre, Lorenzo. Elementos de relatividad. Editorial Universidad de Antioquia. Medellín, 2008.

Einstein, Albert. El significado de la relatividad.
Espasa - Calpe. Madrid, 2008.

Feynman, Richard. QED: La teoría extraña de la luz y de la materia. Prensa de la Universidad de Princeton. Princeton, 1988.

Flores Quelopana, Gustavo. La teoría cosmológica del neutrovacío. Revista Tradición, Año XIV, $\mathrm{N}^{\circ}$ 14, Universidad Ricardo Palma, URP. Lima, 2014.

Hawking, Stephen. Historia del tiempo. Editorial Grijalbo, S.A. Bogotá, 1989. 